\title{
Recycling of Used Hydrogenated Nitrile Butadiene Rubber Through Powder Modification by Trans-Polyoctylene Rubber
}

\author{
Guiying Zhao*, Zhongguang Wang, Taichuang Liu, Xiaoping Zhang
}

Xuzhou College of Industrial Technology, Xuzhou 221140, China

Corresponding Author Email: zhaoguiying2008@163.com

https://doi.org/10.18280/acsm.430604

Received: 3 May 2019

Accepted: 14 September 2019

\section{Keywords:}

hydrogenated nitrile butadiene rubber (HNBR), oil-resistant rubber hose, transpolyoctylene rubber (TOR), rubber powder, modification, recycling

\begin{abstract}
This paper mainly explores the modification of hydrogenated nitrile butadiene rubber (HNBR) powder with trans-polyoctylene rubber (TOR), and the application of the modified HNBR powder in the rubber lining of oil-resistant HNBR hose. The HNBR powder modified by the TOR was prepared, and different dosages of modified powder were filled into the HNBR lining. The resulting specimens were subjected to compression test, aging test, medium resistance test, etc. The results show that the rubber filled with modified powder always outperformed the rubber filled with original powder, indicating that the TOR improves the compatibility between rubber and powder; With the growing dosage of rubber powder, the rubber compound saw a decline in hardness, tensile strength, elongation and tear strength, but a rise in compression set; The aged rubber compound had a greater hardness and tensile strength, but a smaller elongation than the original rubber compound; The cost effectiveness of the rubber compound was maximized when 15 parts of rubber powder were added; In this case, all physical properties were all far above the standard for the rubber lining of oilresistant rubber hoses. The research results help to promote the recycling of used rubber and improve the environment.
\end{abstract}

\section{INTRODUCTION}

The rapid development of the automotive industry has raised higher requirements on the rubber accessories for automobiles. The rubber lining is a key part of oil-resistant rubber hoses in automobiles. However, the traditional material of the rubber lining, nitrile-butadiene rubber (NBR), has many defects, and is being gradually replaced with special rubbers like the hydrogenated nitrile butadiene rubber (HNBR). The HNBR is a highly saturated elastomer obtained through special hydrogenation of the NBR. This type of special rubber enjoys various advantages, ranging from good heat resistance, high strength to small pressure change. Thanks to its excellent overall performance, the HNBR has been widely adopted for the O-rings, seals and hoses of automobile engines.

With the growing use of HNBR vulcanizates, there is a steady increase in used HNBR. The recycling of used HNBR is an imminent task. But no report in China has explored deep into this issue. An important way to recycle used rubbers is to grind them into rubber powder. Unlike the rubber surface, the surface of rubber powder is inert. Therefore, the rubber powder is not highly compatible with rubber. If mixed directly, it is difficult for the two materials to form a good interface. Hence, the surface of rubber powder should be modified to improve the bonding and dispersion effects of the interface.

The trans-polyoctylene rubber (TOR) is a partially crystalline rubber. The unsaturated double bonds in its molecular structure can participate in the crosslinking reaction of rubber. In rubber processing, the TOR serves as a compatibilizer that improves the affinity between different rubbers, and promotes the dispersion of the filler in the rubber [1-4]. Ismail and Akil [5] applied the TOR in the blends of polypropylene (PP)/ethylene-propylene-diene terpolymer (EPDM)/natural rubber (NR), and discovered that the addition of the TOR made the blends more homogenous and improved the adhesion between different phases. Noriman et al. [6] explored how the addition of the TOR affects the properties of styrene butadiene rubber/recycled acrylonitrile butadiene rubber (SBR/NBRr) blends.

Kader et al. [7] and Nah [8] studied the impacts of the TOR on the morphology, dynamic mechanical properties and tensile properties of natural rubber NR/NBR blends. Munirah et al. [9] investigated the impacts of the TOR on the properties and expansion behavior of activated carbon filled SBR vulcanizates, revealing that compatibilized SBR vulcanizate has a shorter curing time and a lower crosslink density than the uncompatibilized SBR vulcanizate, and that its crosslink density slightly decreased with the growing amount of the TOR. Dahham et al. [10] applied the TOR as a compatibilizer in epoxidized natural rubber/recycled silicone catheter (ENR25/RSC) vulcanizate, concluding that the TOR improved the overall performance of ENR-25/RSC vulcanizate, and that the tensile and physical properties of the vulcanizate are both positively correlated with the TOR content.

Dahham et al. [11] observed the following phenomena after using different TOR contents $(2,4,6,8$ and 10 parts) in sawdust (SD) flour reinforced ENR-25: the rising TOR content reduced scorch time and curing time, as well as the viscosity of the rubber compound, which is conducive to processing; a high TOR content (10 parts) brought a stronger interface adhesion between SD and ENR-25, thus improving the tensile strength and modulus of the rubber compound. Dahham et al. [12] investigated the influence of the TOR over the rubber compound performance of nitrile-butadiene 
rubber/natural rubber latex gloves (NBR/NRL-G); the results show that, compared with the uncompatibilized NBR/NRL-G, the compatibilized NBR/NRL-G compound has good curing features, morphology and physical properties; the tensile strength of the compound is the best when the TOR dosage is smaller than 6 parts.

Azimi et al. [13] examined how the TOR affects the curing features and morphological properties of styrene butadiene rubber/recycled chloroprene rubber (SBR/CRr) blends. Azrem et al. [14] investigated the effects of the TOR on the physical and morphological properties of the SBR/CRr blends. Awang and Ismail [15] studied the impacts of the TOR and dynamic curing on the weatherability of polypropylene/waste tire powder blends. Noriman et al. [16] probed into the influence of the TOR addition on the properties of SBR/NBR blends. Dahham et al. [17] deliberated the effects of wood flour and the TOR on the performance of the NBR filler in the NRL gloves. Noriman et al. [18] studied the influence of the TOR on thermal and fatigue performance of SBR/recycled NBR. Awang et al. [19] explored the impacts of the TOR and dynamic curing on the properties of polypropylene-based blends containing waste tire rubber powder.

The above studies on the application of the TOR in rubbers mainly focus on enhancing the compatibility between rubbers through TOR modification, thus improving the processing physical properties of the rubber compound. However, none of them tackles the modification of the power of used rubber. To make up for the gap, this paper mechanically pulverizes used HNBR into rubber powder, and modifies the surface of the powder with the TOR, making the powder more compatible with rubber. In addition, the author explored how the dosage of the modified powder affects the performance of the rubber lining in oil-resistant HNBR hose. The research comes up with a rational method to modify the powder of used HNBR, and the reasonable dosage of the modified powder in oil-resistant HNBR hose, laying a good basis for the recycling of used HNBR vulcanizates.

\section{EXPERIMENTS}

\subsection{Materials and instruments}

The following raw materials were selected for our experiments: HNBR-4307 (LANXESS, Germany); TOR, VESTENAMER 8012 (Evonik China); carbon black, N550 (Jiangxi Black Cat Carbon Black Co., Ltd., China); zinc oxide, stearic acid, dicumyl peroxide (DCP), triallyl isocyanurate (TAIC), age resister and plasticizer were all purchased from the market.

The following instruments were adopted for our experiments: XK-160 open mixing mill and QLB-50D/Q flat vulcanizer (Wuxi No.1 Rubber \& Plastics Mechanical Co., Ltd., China) for rubber compounding and curing; GT-M2000A rotorless vulcanizer and computer-controlled tensile tester (Taiwan High Speed Rail Corporation, China) for measuring processing and mechanical properties; 401-B aging oven (Shanghai Laboratory Instrument Co., Ltd., China) for testing the properties of aging resistance and medium resistance.

\subsection{Preparation of modified powder}

(1) Mechanical pulverization: Freeze the used HNBR vulcanizate at low temperature for $5 \mathrm{~h}$, making it hard and brittle. Reduce the roller distance of the open mixing mill to $0.2 \mathrm{~mm}$. Put the waste HNBR vulcanizate into the open mill for 10 to 20 times. Mechanically pulverize the waste HNBR vulcanizate into rubber powder. The number of rolling depends on the specific situation. Be careful not to wrap the rolls to prevent sheeting.

(2) Surface modification: Heat up the open mixing mill to about $70^{\circ} \mathrm{C}$, and then add the TOR (the modifier) to melt at the high temperature. Next, add the prepared rubber powder, and mix the modifier tor and rubber powder through the roller 8 to 10 times. In this way, the surface of the rubber power is modified, creating the modified HNBR powder. The dosage of the modifier is $5 \%$ of the total mass of the rubber powder.

\subsection{Composition of rubber compound}

The composition (parts by mass) of rubber compound is as follows: stearic acid, 1; zinc oxide, 4; age resister, 1.5; DCP, 3; TAIC, 2; epoxy soybean oil, 10; HNBR powder (variable) $0,5,10,15,15,20$ and 25 . The powder is not modified only in the third formula.

\subsection{Sample preparation}

Preparation of compound rubber: Adjust the roller distance to $0.1 \mathrm{~mm}$, perform thin-pass plasticizing of the HNBR 3 to 4 times, and then increase the roller distance. Then, add stearic acid, zinc oxide and age resister in turn. After that, add carbon black, plasticizer and rubber powder, and adjust the roller distance to $2.4 \mathrm{~mm}$ once the materials are all added. Then, add vulcanizing agent, and auxiliary agent, and making 5 triangle bags through thin pass and 3 big rolls. Finally, increase the roller distance and obtain the rubber compound.

Primary curing: Let the rubber compound stand for a specified period, and then test its process performance. After that, vulcanize each sample on the flat vulcanizer at $170^{\circ} \mathrm{C}$ for T90.

Secondary curing: Let the primarily cured sample stand for a specified period, and then relocate it into the aging oven for secondary curing at $165^{\circ} \mathrm{C}$ for $4 \mathrm{~h}$. The secondary curing aims to improve the crosslinking of the rubber products, enhancing the overall physical and mechanical properties of the rubber compound.

\subsection{Performance testing}

Each performance parameter was measured as per the corresponding national standard. Specifically, the medium resistance test uses engine oil, the compression test uses type $\mathrm{B}$ specimens, and aging resistance, medium resistance and compression tests were conducted at $150^{\circ} \mathrm{C}$ for $72 \mathrm{~h}$ each.

\section{EXPERIMENTAL RESULTS AND DISCUSSION}

\subsection{Influence of rubber powder content on the processing performance of HNBR inner rubber}

Table 1 shows the variation in processing performance of HNBR inner rubber with the dosage of rubber powder. 
Table 1. Influence of rubber powder content on the processing performance of HNBR inner rubber

\begin{tabular}{cccccc}
\hline Curing parameters & $\begin{array}{c}\text { Maximum } \\
\text { torque } \\
\text { Rubber powder content }\end{array}$ & $\begin{array}{c}\text { Minimum } \\
\text { torque } \\
\text { (ML) dN·m }\end{array}$ & $\begin{array}{c}\text { Time to 10\% } \\
\text { torque increase } \\
\text { (tc10) m: } \mathbf{s}\end{array}$ & $\begin{array}{c}\text { Time to 90\% } \\
\text { torque increase } \\
\text { (tc90) m: } \mathbf{s}\end{array}$ & $\begin{array}{c}\text { Time to 100\% } \\
\text { torque increase } \\
\text { (tc100) } \mathbf{~ m : ~} \mathbf{s}\end{array}$ \\
\hline Modified powder-0 & 28.42 & 4.05 & $0: 55$ & $8: 05$ & 19 \\
Modified powder-5 & 28.63 & 3.10 & $1: 05$ & $8: 23$ & $17: 02$ \\
Modified powder-10 & 28.50 & 4.10 & $1: 03$ & $8: 15$ & $19: 37$ \\
Original powder-15 & 24.05 & 3.90 & $1: 01$ & $8: 00$ & $16: 52$ \\
Modified powder-15 & 28.05 & 4.17 & $1: 02$ & $8: 10$ & $19: 17$ \\
Modified powder-20 & 25.28 & 4.44 & $0: 58$ & $8: 04$ & $19: 06$ \\
Modified powder-25 & 24.13 & 4.49 & $1: 00$ & $8: 14$ & $19: 17$ \\
\hline
\end{tabular}

As shown in Table 1, with the addition of rubber powder, the scorch time and the optimum curing time of the rubber compound slightly increased, which improves the operating safety. Hence, a proper amount of rubber powder has little effect on the curing time of the rubber compound. Moreover, the $\mathrm{MH}$ of the rubber compound slightly increased, when 5 or 10 parts of rubber powder were added. Further growth in the dosage of rubber powder suppressed the $\mathrm{MH}$ and rigidity of

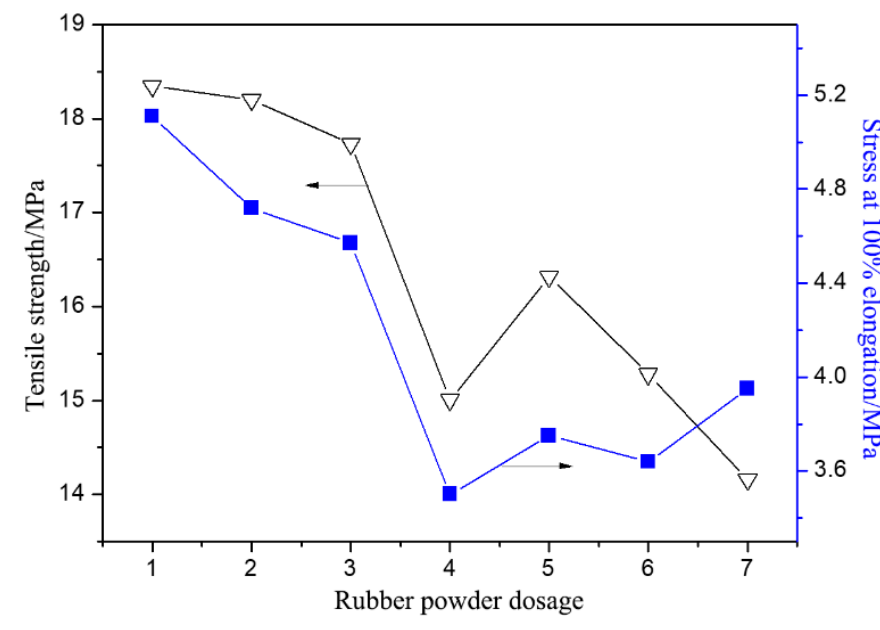

Figure 1. The tensile strength, stress at 100\% elongation, hardness and elongation of rubber compound

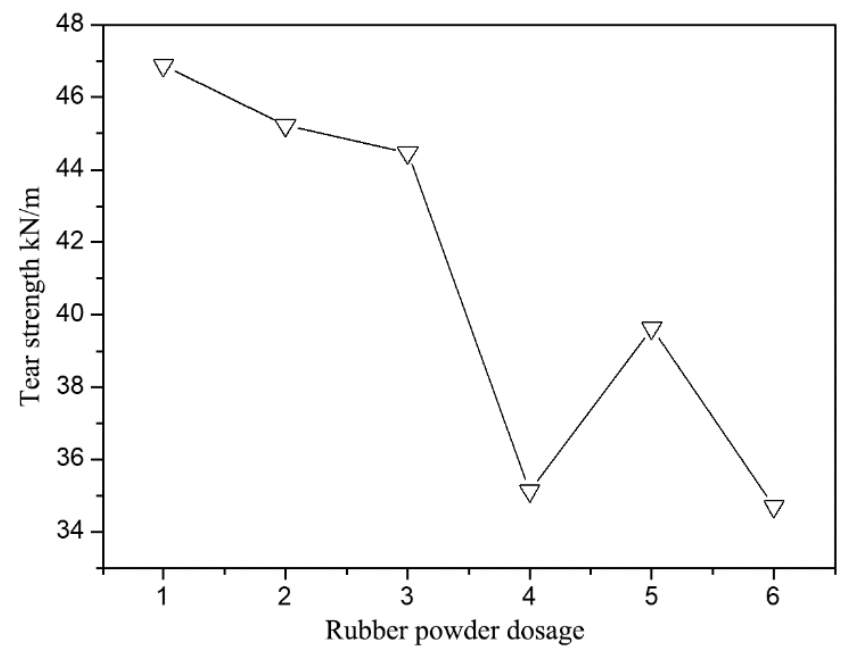

Figure 2. Tear strength of rubber compound

As shown in Figures 1 and 2, with the growing dosage of rubber powder, the tensile strength, stress at $100 \%$ elongation, maximum elongation and tear strength of the rubber compound were all declining. When the rubber powder content reached 25 parts, the hardness, tensile strength,

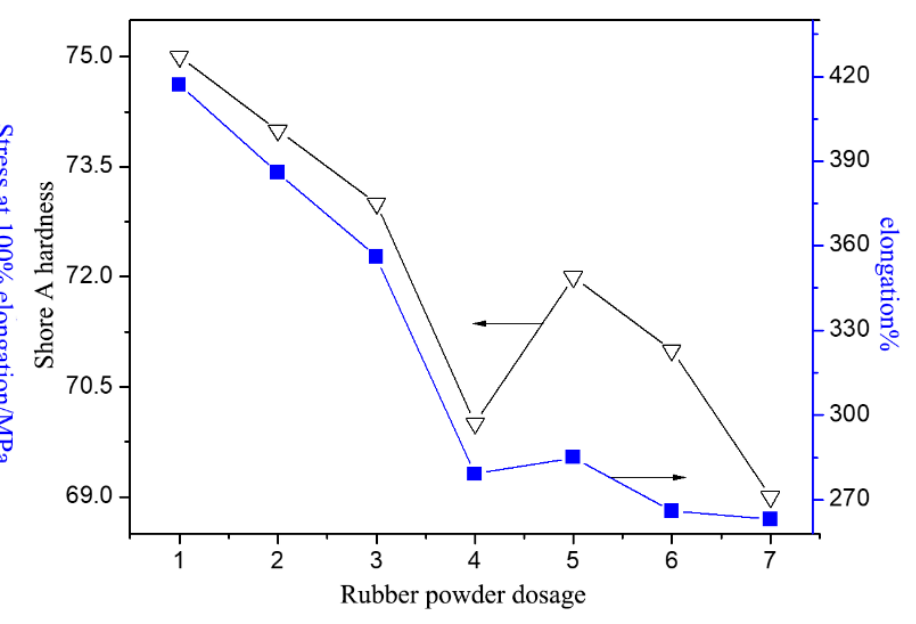

the rubber compound.

\subsection{Influence of rubber powder content on the mechanical performance of HNBR inner rubber}

Figures 1 and 2 display the variation in the mechanical performance of HNBR inner rubber with the dosage of rubber powder.

elongation and tear strength of the rubber compound were minimized, respectively at 75 (Shore A hardness), 14.16MPa, $263 \%$ and $33.32 \mathrm{kN} / \mathrm{m}$.

Then, the performance of the rubber filled with modified powder was compared with that of the rubber filled with original powder at the same dosage. It can be seen that the rubber filled with the original powder $4 \#$ showed much smaller hardness, tensile strength, elongation and tear strength than the rubber filled with modified powder $5 \#$. The possible reasons are as follows: after surface modification by the TOR, the TOR on the surface of rubber powder takes part in the crosslinking reaction during the curing process, linking the powder and the rubber together and promoting the compatibility between the two entities. Therefore, the modified powder can enhance the strength of the rubber. By contrast, the original powder is not well dispersed in the rubber, failing to improve the rubber strength.

\subsection{Influence of rubber powder content on the aging resistance of HNBR inner rubber}

Figure 3 illustrates the variation in the aging resistance of HNBR inner rubber with the dosage of rubber powder. 

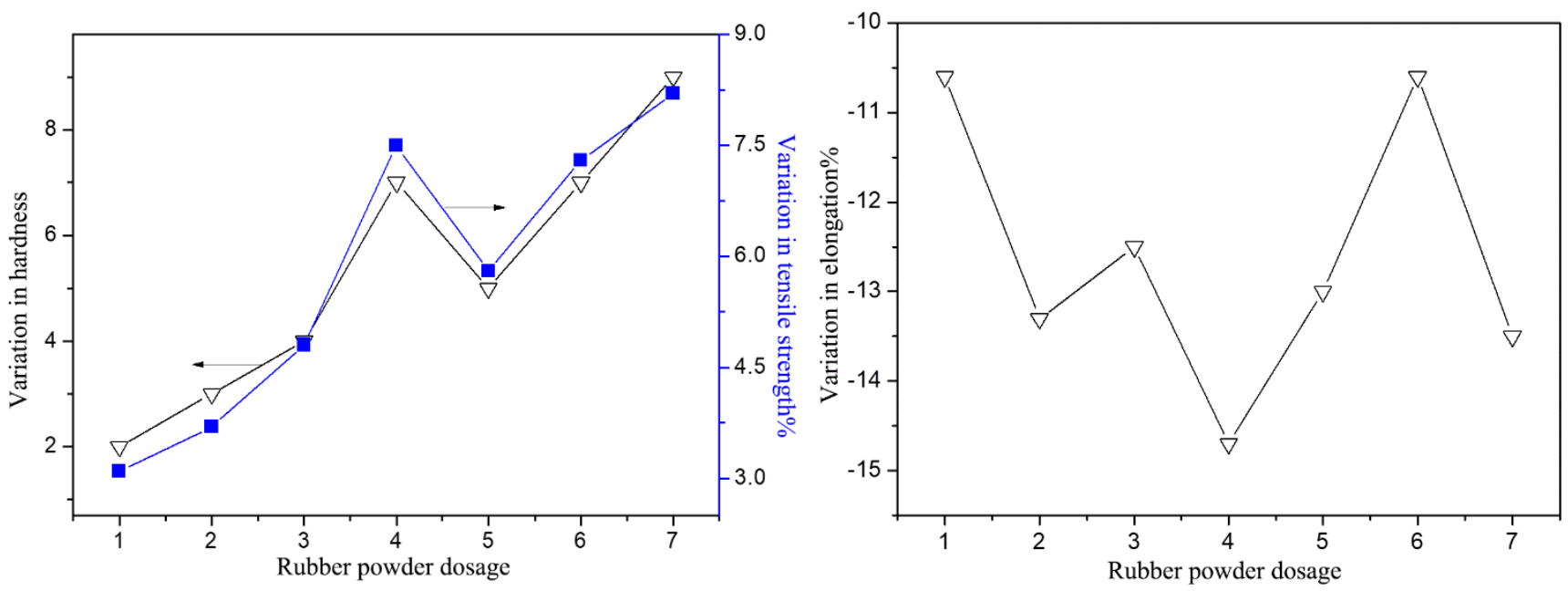

Figure 3. Variations in hardness, tensile strength and elongation of the aged rubber compound

As shown in Figure 3, the rubber compound had a greater tensile strength after aging. Under the aging conditions, the rubber compound was post-cured, which enhances the crosslink network. The rubber filled with 25 parts of rubber powder experienced the greatest variation in tensile strength.

The aging process reduced the elongation of the rubber compound. The rubber filled with the original powder suffered the largest decrease in elongation $(-14.7 \%)$, and the rubber not filled with any rubber powder saw the smallest decrease in elongation $(-10.6 \%)$.

Through the aging process, the hardness of the rubber compound increased across the broad. The hardness is positively correlated with the dosage of rubber powder.

Overall, the aging resistance of the rubber compound did not change greatly, when the dosage of rubber powder was smaller than 15 parts.

\subsection{Influence of rubber powder content on the medium resistance and compressive strength of HNBR inner rubber}

Figure 4 provides the variation in the medium resistance and compressive strength of HNBR inner rubber with the dosage of rubber powder.
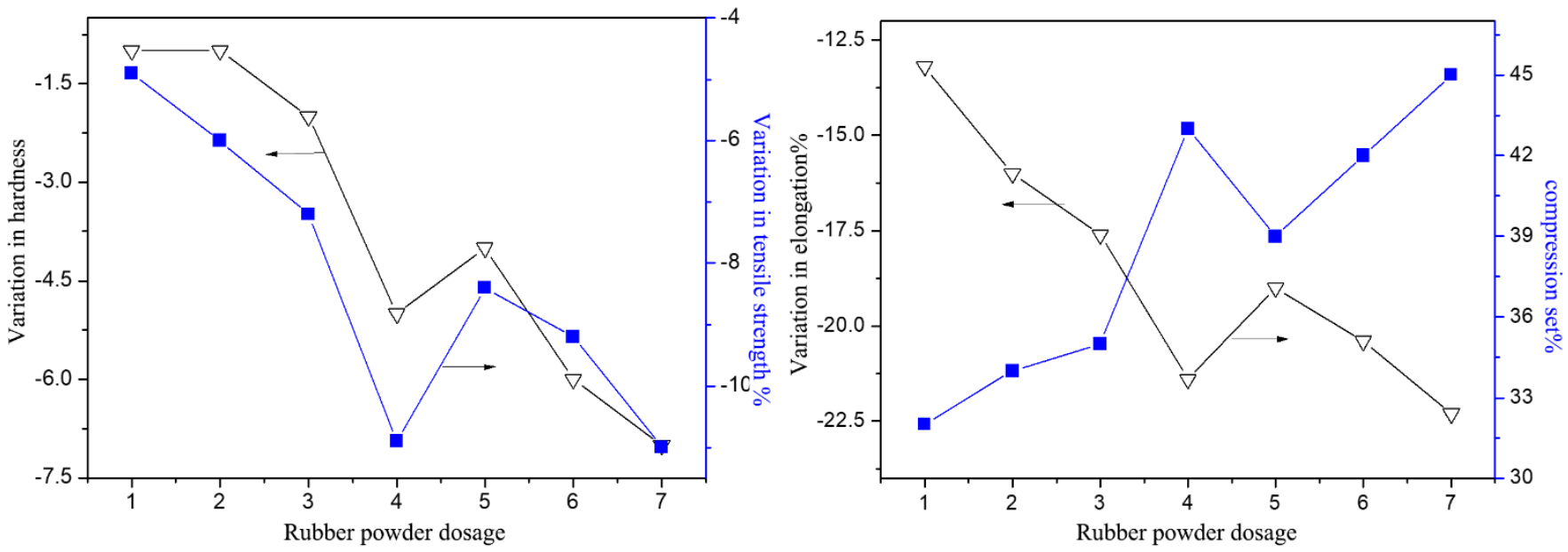

Figure 4. Variations in hardness, tensile strength, elongation and compression set of the rubber compound after the medium resistance test

As shown in Figure 4, through the medium resistance test, the rubber compound had a decline in hardness, tensile strength and elongation. With the growth in rubber powder content, these three mechanical properties decreased steadily. This is because the plasticizer and active substance in the formula and rubber powder are extracted by the oil, causing damages to the internal structure. In general, the medium resistance of the rubber compound remained basically stable, when fewer than 15 parts of modified powder were filled into the rubber. The rubber filled with modified powder was more resistant to oil medium than the rubber filled with the original powder.
It can also be seen from Figure 4 that the HNBR rubber compound with no powder had the smallest compression set $(32 \%)$, and the rubber compound filled with 25 parts of powder had the largest compression set (45\%). The higher the powder dosage, the weaker the compressive strength of the rubber compound.

\section{CONCLUSIONS}

(1) With the addition of rubber powder, the scorch time and the optimum curing time of the rubber compound slightly 
increased, which improves the operating safety. Moreover, the $\mathrm{MH}$ of the rubber compound slightly increased, when 5 or 10 parts of rubber powder were added. Further growth in the dosage of rubber powder suppressed the $\mathrm{MH}$ and rigidity of the rubber compound.

(2) With the growing dosage of rubber powder, the rubber compound saw a decline in hardness, tensile strength, elongation and tear strength, but a rise in compression set. The aged rubber compound had a greater hardness and tensile strength, but a smaller elongation than the original rubber compound. After the high-temperature oil resistance test, the hardness, tensile strength and elongation of the rubber compound all decreased. The rubber filled with modified powder always outperformed the rubber filled with original powder, indicating that the TOR improves the compatibility between rubber and powder.

(3) The cost effectiveness of the rubber compound was maximized when 15 parts of rubber powder were added. In this case, the hardness, tensile strength, elongation, tear strength and compression set of the rubber compound were 72 , $16.32 \mathrm{MPa}, 39.63 \mathrm{kN} / \mathrm{m}, 285 \%$ and $39 \%$, respectively; through aging, the tensile strength, elongation and hardness of the rubber compound changed by $+5.8 \%,-13 \%$ and +5 , respectively; after the oil resistance test, the tensile strength and elongation of the rubber compound changed by $-8.4 \%$ and $-19 \%$, respectively.

\section{ACKNOWLEDGMENT}

This paper is supported by Scientific Research Project of Xuzhou Municipality 2016 (Grant No.: KC16SG271).

\section{REFERENCES}

[1] Nan, C., Han, S.C., Jo, B.W., Kim, W.D. (2002). Influences of trans-polyoctylene rubber on the Physical Properties and Phase morphology of natural rubber /acrylonitrile-butadiene rubber blends. Journal of Applied Polymer Science, 86(1): 125-134. https://doi.org/10.1002/app.10827

[2] Fatin, M.H., Zulkepli, N.N., Sam, S.T., Zakaria, M.S. (2014). The effects of trans-polyoctyene rubber (TOR) as compatibilizer on recycled nitrile glove (NBRr) filled natural rubber compound: Tensile test and morphology studies. Applied Mechanics and Materials, 305-310. https://doi.org/10.4028/www.scientific.net/AMM.679.3 05

[3] Commereucs, S., Lacoste, J. (1997). Photo-and thermooxidation of polyoctenamer. photostability of hydroperoxides. Polymer Degradation and Stability, 57(1): $\quad 31-41 . \quad$ https://doi.org/10.1016/S01413910(96)00225-X

[4] Chattaraj, P.P., Kalidaha, A.K., Mukhopadhyay, R., Bhattacharya, A.K., Tripathy, D.K. (2006). Rheological study of filled SBR compounds with transpolyoctenylene (TOR) and their interaction mechanism. International Journal of Polymeric Materials and Polymeric Biomaterials, 33(1-2): 73-87. https://doi.org/10.1080/00914039608028609

[5] Ismail, H., Akil, H.M. (2005). The effects of transpolyoctene rubber and dynamic vulcanization on properties of PP/EPDM/NR blends. Progress in Rubber
Plastics Recycling Technology, 21(4): 39-53. http://dx.doi.org/10.1177/147776060502100103

[6] Noriman, N.Z., Ismail, H., Rashi, A.A. (2012). Properties of styrene butadiene rubber/recycled acrylonitrilebutadiene rubber (SBR/NBRr) blends: Effect of the addition of trans-polyoctylene rubber. Journal of Applied Polymer Science, $126 \quad$ (S2): $\quad$ E56-E63. http://dx.doi.org/10.1002/app.36402

[7] Kader, M.A., Kim, W.D., Kaang, S., Nah, C.D. (2005). Morphology and dynamic mechanical properties of natural rubber/nitrile rubber blends containing transpolyoctylene rubber as a compatibilizer. Polymer International, $\quad$ 54(1): $120-129$. https://doi.org/10.1002/pi.1655

[8] Nah, C. (2002). Effects of trans-polyoctylene rubber on Rheological and green tensile properties of natural rubber/acrylonitrile-butadiene rubber blends. Polymer International, $\quad 51(3)$ : 245-252. https://doi.org/10.1002/pi.851

[9] Munirah, N.R., Noriman, N.Z., Salihin, M.Z., Sam, S.T., Al Bakri M.M.A., Hussin, K., Rosniza, H., Omar, M.F. (2016). The effects of trans-polyoctylene rubber (TOR) on the cure characteristics and swelling behaviour of activated carbon filled styrene butadiene rubber (SBR) vulcanizates. Materials Science Forum, 857: 164-168. https://doi.org/10.4028/www.scientific.net/MSF.857.16 4

[10] Dahham, O.S., Noriman, N.Z., Sam, S.T., Rosniza1, H., Al-Samarrai Marwa, N., Shayfull, Z., Alakrach, A. M. (2016). The effects of trans-polyoctylene rubber (TOR) as a compatibilizer on the properties of epoxidized natural rubber/recycled silicone catheter (ENR-25/rSC) Vulcanizate. MATEC Web of Conferences, 78(01076). https://doi.org/10.1051/matecconf/20167801076

[11] Dahham, O.S., Zulkepli, N.N., Hamzah, R., Idrus, S.Z.S., Rahim, S.Z.B.A., Adam, T., Jaya, H. (2018). Sawdust flour reinforced epoxidized natural rubber: Effect of trans-polyoctylene rubber on curing characteristics and tensile properties. Journal of Physics, 1019(1): 012090. https://doi.org/10.1088/1742-6596/1019/1/012090

[12] Dahham, O.S., Noriman, N.Z., Husin, K., Sam, S.T., Omar, M.F., Mustafa Al Bakri, A.M., Rosniza, H. (2015). The effects of trans-polyoctylene rubber (TOR) on the properties of acrylonitrile butadiene rubber/recycled natural latex gloves (NBR/NRL-G) compounds. Applied Mechanics and Materials, 815: 59-63. http://dx.doi.org/10.4028/www.scientific.net/AMM.815 .59

[13] Azmi, A.A., Zulkepli, N.N., Razif, M.N., Sam, S.T., Abdullah, M.M.A.B. (2013). The influences of tor as a compatibilizer on cure characteristic and morphological properties of SBR/CRr blends. Advances in Environmental Biology, 7(12): 3743-3746.

[14] Azrem, A.A., Zulkepli, N.N., Razif, M.N., San, S.T. (2014). Physical and morphological properties of styrene butadiene rubber/recycled chloroprene rubber (SBR/CRr) blends, The effects of TOR as a compatibilizer. Key Engineering Materials, 594: 862-866. http://dx.doi.org/10.4028/www.scientific.net/KEM.594595.862

[15] Awang, M., Ismail, H. (2009). Weatherability of polypropylene/waste tire dust blends, Effects of transpolyoctylene rubber and dynamic vulcanization. Journal of Vinyl and Additive Technology, 15(1): 29-38. 
https://doi.org/10.1002/vnl.20172

[16] Noriman, N.Z., Ismail, H., Rashid, A.A. (2012). Properties of styrene butadiene rubber/recycled acrylonitrile-butadiene rubber (SBR/NBRr) blends, Effect of the addition of trans- polyoctylene rubber. Journal of Applied Polymer Science, 126(S2): 56-63. https://doi.org/10.1002/app.36402

[17] Dahham, O.S., Zulkepli, N.N., Sam S.T., Ismail, H., Santiagoo, R., Hamzah, R., Al-samarrai, M. (2016). Properties of recycled natural latex gloves filled NBR: Effects of sawdust and trans polyoctylene rubber. Journal of Polymer Materials, 33(4): 647-655.
[18] Noriman, N.Z., Ismail, H., Rashid, A.A. (2011). The Effects of trans-polyoctylene rubber on thermal analysis and fatigue properties of styrene butadiene rubber/recycled acrylonitrile-butadiene rubber. Advances in Polymer Technology, 31(2): 100-108. http://dx.doi.org/10.1002/adv.20240

[19] Awang, M., Hazizan, M., Akil, H., Ismail, H. (2007). Polypropylene-based blends containing waste tire dust: Effects of trans-polyoctylene rubber (TOR) and dynamic valcanization. Polymer testing, 26(6): 779-787. http://dx.doi.org/10.1016/j.polymertesting.2007.04.007 